\title{
Functional transplant of megabase human immunoglobulin loci recapitulates human antibody response in mice
}

Mendez, Michael J. et al. Nature Genet. 15, 146-156 (1997).

The following is a correction to authorship:

Michael J. Mendez ${ }^{2}$, Larry L. Green ${ }^{1}$, Jose R.F. Corvalan ${ }^{1}$, Xiao-Chi Jia ${ }^{1}$, Catherine E. Maynard-Currie ${ }^{1}$, Xiao-dong Yang ${ }^{1}$, Michael L. Gallo ${ }^{1}$, Donna M. Louie ${ }^{1}$, Doris V. Lee ${ }^{1}$, Karen L. Erickson ${ }^{1}$, Jac Luna ${ }^{2}$, Catherine M.-N. Roy ${ }^{1}$ Hadi Abderrahim $^{2}$, Ford Kirschenbaum ${ }^{2}$, Masato Noguchi², Douglas H. Smith², Atsushi Fukushima ${ }^{2}$, Joanna F. Hales ${ }^{1}$, Sue Klapholz ${ }^{2}$, Mitchell H. Finer ${ }^{2}$, C. Geoffrey Davis ${ }^{1}$, Krisztina M. Zsebo ${ }^{2}$ \& Aya Jakobovits ${ }^{1}$

${ }^{1}$ Abgenix, Inc., 7601 Dumbarton Circle, Fremont, California 94555, USA. ${ }^{2}$ Cell Genesys, Inc., Foster City, California 94404, USA

\section{erratum}

\section{Regulation of cardiac mesodermal and neural crest development by the bHLH transcription factor, dHAND}

Deepak Srivastava et al. Nature Genet. 16, 154-160 (1997).

Due to a printing error, the contrast between colour shades in Fig. 1 was suboptimal, obscuring the difference in ventricular expression of dHAND (Fig. 1c).
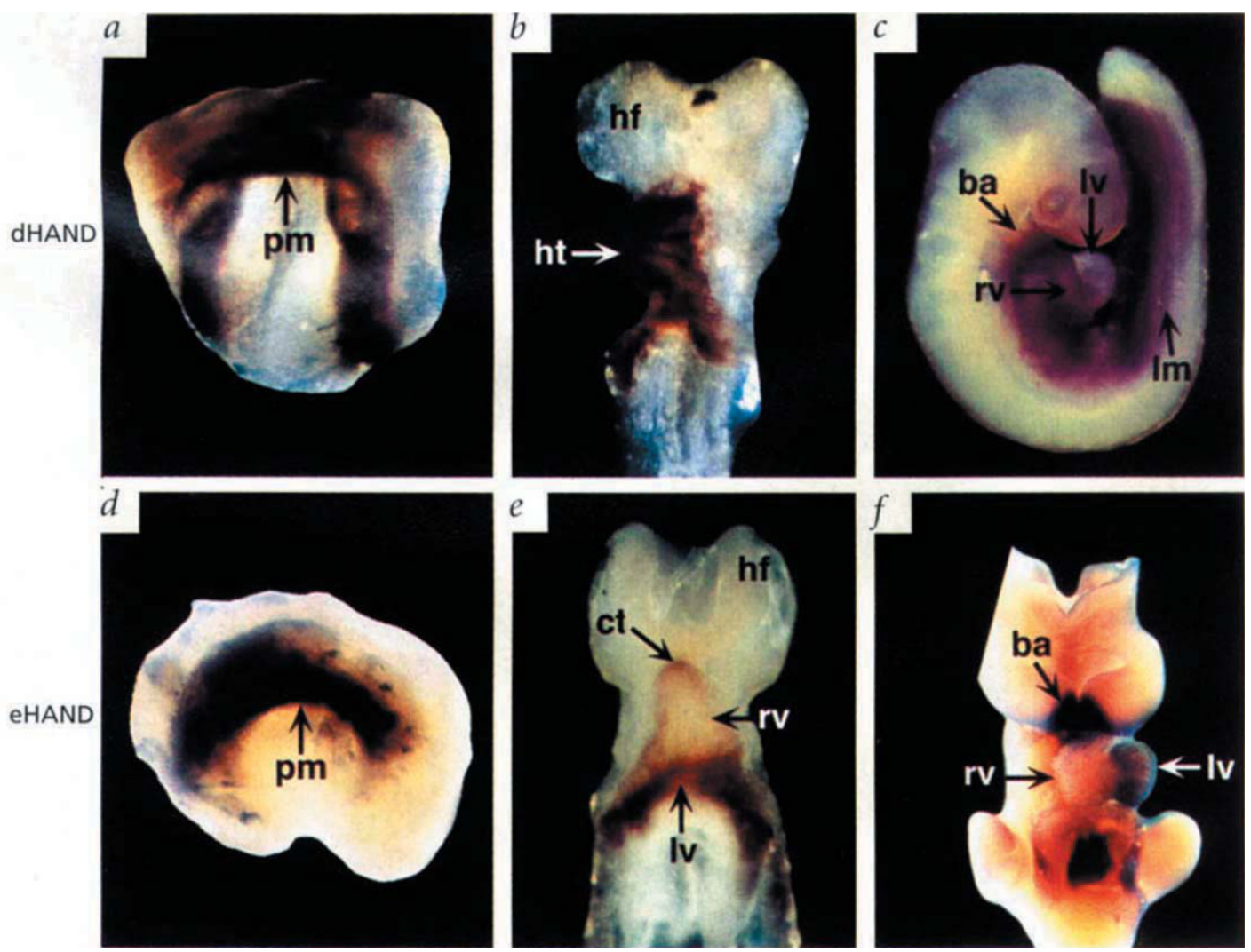

Fig. 1 Expression of dHAND and eHAND mRNA in staged mouse embryos by whole mount in situ hybridization. Panels show embryos at the following stages: $\boldsymbol{a}, \boldsymbol{d}, E 7.75 ; \boldsymbol{b}, \mathbf{e}, \mathrm{E} 8.0 ; \boldsymbol{c}, E 9.5 ; \boldsymbol{f}, \mathrm{E10.0}$. In e, note that eHAND expression is restricted to the anterior and posterior segments of the heart tube, but is symmetric along the left-right axis of the embryo. Note that dHAND expression is restricted to the right and eHAND to the left side of the developing heart ( $c$ and $f$, respectively). pm, precardiogenic mesoderm; Im, lateral mesoderm; ht, heart tube; ct, conotruncus; rv, right ventricle; lv, left ventricle; ba, branchial arch; hf, head fold. 